\title{
Conjugación del talento humano y la gestión pública: de lo político, lo público, lo institucional, lo estratégico
}

\section{Conjugation of the human talent and the public management: of the political, the public, the institutional, the strategic}

\author{
Yoselin García Colina \\ yoselyn3110@gmail.com
}

Gobernación Bolivariana del Estado Monagas

Venezuela

Recibido: 30 de junio de 2017

Aprobado: 25 de julio 2017

\section{RESUMEN}

El talento humano constituye un factor fundamental para el desarrollo de la institucionalidad venezolana, ya que interviene de manera directa en el seguimiento a las políticas de un sistema de gobierno y que incide en la gestión pública. Se hace necesario políticas que transfiguren la gestión del personal desde la formalidad institucional enmarcada en el proceso social de trabajo, para responder a las demandas de las comunidades, resulta una tarea obligada la elaboración y ejecución de modelos organizacionales que respondan a la gestión de talento humano de una manera integrada, sistémica y estrechamente vinculada entre las necesidades colectivas de desarrollo y las individuales de crecimiento, para lograr mejores profesionales formados bajo este paradigma de gestión emergente, contribuyendo a satisfacer las necesidades como motor en la administración y distribución de los recursos del estado y que se determinen por la capacidad de respuesta de los entes que conduzca hacia el desarrollo.

Palabras clave: Talento humano, gestión pública, política pública. 


\begin{abstract}
The human talent is a very important factor for developing in Venezuelan institutions, due to it is joined in the direct way to follow policies of a government system, and of course it intervenes in public management. So it's necessary to have policies that transfer the personal management to the formal institutional policies in working social process to respond to the communities requesting. It results in a target in making and executing organizational models to respond the human talent management in an integrative, systemic and closely linked between developing collective needs and individual growth needs, to get the best professionals formed under this management paradigm to contribute for satisfying institutional needs, like the engine in the administration and distribution in the state wealth, determined by the abilities to moves forward the development in the frame of its potentialities.
\end{abstract}

Key words: Human talent, public management, public policies.

\title{
INTRODUCCIÓN
}

En el marco del proceso de transformación que el estado venezolano ha impulsado en función del desarrollo del poder popular, de las organizaciones y movimientos sociales que forman parte de un conjunto de acciones sustentadas en los elementos que orbitan alrededor de los ideales Bolivarianos y que a groso modo destaca como sistema de gobierno a la democracia protagónica y participativa que permitan la vinculación activa del pueblo en los planes, programas y proyectos encaminados a procurar el crecimiento económico, social, cultural y político de las comunidades.

Es donde la participación como forma de acción social para encaminar las decisiones gubernamentales relacionadas a las políticas públicas de solución y resolución de situaciones que ponen en desventaja a un grupo social, ha permitido transferir iniciativas colectivas del ejercicio del poder en tanto en cuánto se ha hecho praxis de las formas de administración de los recursos del tesoro del estado a manos de comunidades organizadas que intervienen de manera directa, desde la jerarquización de las necesidades hasta la ejecución de obras, que permiten la sinergia de la 


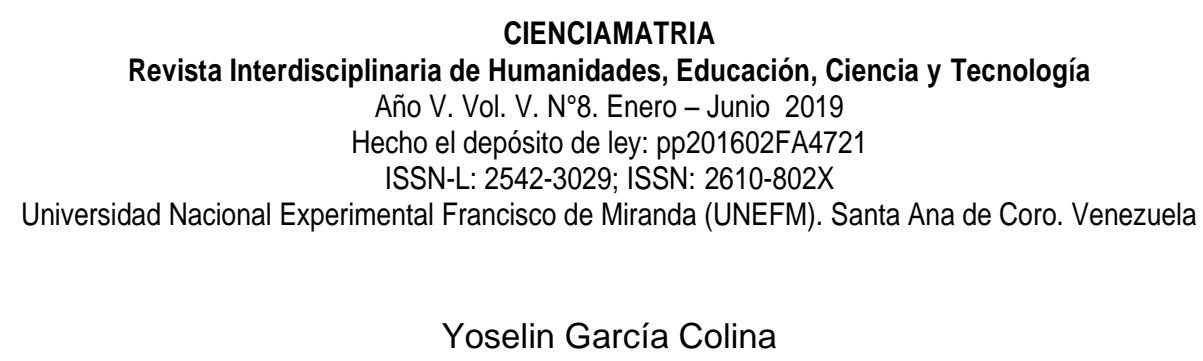

colaboración institucional y un nuevo sistema de gobierno popular orientado así en la Ley del Plan de la Patria (2013).

En este sentido, resulta menester destacar los grandes avances que, sin lugar a dudas, ha reconocido a la clase trabajadora no sólo en reivindicaciones salariales, sino también en el reconocimiento de la fuerza motora que refiere, en este caso, a las y los trabajadores y trabajadoras del sector de la administración pública en lo general.

Sin embargo, este reconocimiento del ser humano como epicentro de las verdaderas transformaciones sociales ha gestado en esa misma medida la exigencia de la formación ética, profesional, política, del actor social que implica un lugar en la estructura de gobierno, que refiere a las diferentes áreas de conocimiento y que son necesarias para el desarrollo y crecimiento de la nación en los espacios de atención fundamental, por ejemplo, la salud, la educación, la seguridad, y en otros términos, infraestructura, administración, etc.

Precisamente, para lograr el perfil del trabajador y trabajadora cónsono con la realidad no sólo generacional, en lo histórico, sino también de manera integral, que cumpla con su responsabilidad en el apalancamiento de la nación, que pueda llegar a ser un factor determinante en el desarrollo de la gestión pública, se hace necesario, casi imprescindible, políticas que transfiguren la formación como política institucional formal enmarcada en el proceso social de trabajo.

Los estudios abordados relacionados con el tema, por lo general, giran en torno a la visión capitalista de la formación, teniendo al ser humano como un valor de cambio reduciéndolos a meros recursos organizacionales que se adicionan como parte de la administración del patrimonio (Prieto, 2013).

Esta nueva visión social de la formación implica reconocer al trabajador o trabajadora como parte del poder popular, integrante de la gesta de los procesos de transformación social e institucional; en este sentido, (Chirino y Lamus de Rodríguez, 2017, p. 113), plantean que "Es opinión de las autoras considerar que usando el pensamiento crítico 


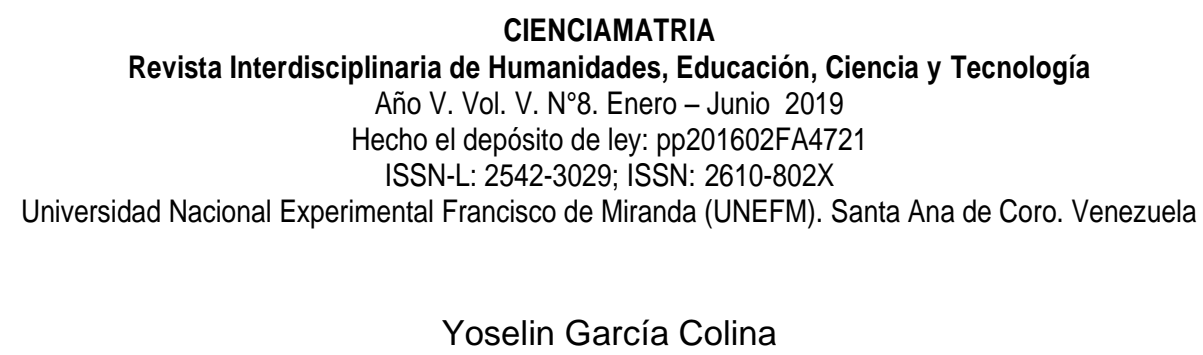

como herramienta de educación popular se puede fortalecer la participación de la comunidad en la toma de decisiones", es necesario tener en consideración que el talento humano en la gestión pública debe estar articulado a una visión crítica reflexiva de su realidad social y política.

resulta menester mencionar que pese a los esfuerzo que ha direccionado como parte de la nueva reorganización del estado, no son suficientes y deben ser abordados de manera más consistente, consecutiva y adecuada a las necesidades y realidades de la gestión relacionada al perfil profesional y la gestión pública en el manejo de la institucionalidad actual.

\section{CONJUGACIÓN DEL TALENTO HUMANO Y LA GESTIÓN PÚBLICA: DE LO POLÍTICO, LO PÚBLICO, LO INSTITUCIONAL, LO ESTRATEGICO}

Las organizaciones públicas y/o privadas a lo largo del tiempo han mantenido un tipo de relación laboral en donde el trato entre empleador y trabajador responde a la concepción mecanicista, en donde lo único importante resulta producir; estas es una lógica tomada de la época en donde la Revolución Industrial tuvo mayor impulso, inclusive en la actualidad existen instituciones universitarias donde los egresados se titulan en "Relaciones Industriales" y que finalmente responden a un sistema de dominación tanto cultural como social.

Posterior a esta etapa burocratizada del manejo y direccionalidad de la fuerza labor, se establece un supuesto cambio de paradigmas al tratar la relaciones entre el que ofrece el trabajo y el que lo recibe como "Administración de Recursos Humanos", en el que se dinamizaba y mediaba entre el trabajo y el que trabajaba, esta visión fue incrementando a lo largo del tiempo y dando giros hacia la "humanización" del poder y del dominio con que se manejaba el recurso dentro de la lógica del capital.

Hoy, a razón del auge globalizado de expresiones de conciencia colectiva que ejerce la clase trabajadora en el mundo, se distinguen diversas formas evolucionadas de 


\section{Yoselin García Colina}

discernir los embates de las estructuras de poder que manejan el poder económico y que en definitiva son los que manejan la economía mundial, esto ha permitido que se erijan nuevas formas de producción y mejoramiento tanto de la calidad de vida como de eficiencia y eficacia en el manejo y puesta en marcha de instituciones y organizaciones. En Venezuela, este proceso se ha dado en gran medida por las políticas legislativas adoptadas en el año 2012 con la Ley Orgánica del Trabajo, los trabajadores y trabajadoras discutida desde las bases y aprobada por decreto por el Presidente Hugo Chávez, en donde se ha reivindicado no solamente los beneficios contractuales, sino también se ha puesto mayor énfasis en el desarrollo humano de los trabajadores y trabajadoras y que, busca la reversión de una causa de empobrecimiento social, además de defender el proceso social del trabajo para apalancar el crecimiento y desarrollo de la nación sustentados en principios morales, éticos e ideológicos.

En este sentido, el desarrollo de la institucionalidad venezolana deviene en gran medida de aspectos que van desde lo económico, legal, cultural, histórico hasta lo social del talento humano que lo conforma. En este sentido, resulta menester puntualizar los elementos que orbitan en torno a la operatividad, funcionabilidad y efectividad de los organismos públicos que sirven de sustento político a un sistema de gobierno.

Nada más lejos del contexto que los entes de gobernanza estadal que son los garantes de propiciar y promover las políticas de estado que erigen las formas de gobierno y la estructura del estado, y que actualmente se encuentran en un momento estático no sólo por su obsolescencia sino también la carga burocrática que implica formas no organizadas ni planificadas en la dirección del personal que dirige los espacios institucionales, y que son parte fundamental para el desarrollo de la organización pública y sobre todo de la política pública que allí se expresa.

Puede resultar atípico o poco ortodoxo, pero para lograr la transformación institucional y procedimental de la misma en función de la eficacia y eficiencia, resulta imperioso el hecho de evaluar como estos dos indicadores se ven permeados por la "mirada 


\section{Yoselin García Colina}

polisémica" de quién maneja la gestión pública. Lo anterior resulta de la diversidad epistémica (Perafán, 2004) que perfila al talento humano como una fuente inagotable de conocimiento producido en gran medida por el empirismo del método de captación, o simplemente, por poseer poca o ninguna formación en el área ocupacional.

El estudio que aquí se procura persigue evidenciar entre otros:

1. ¿Por qué los modelos organizacionales de gestión del talento humano no constituyen un elemento vital para el desarrollo humano e institucional?

2. ¿En cuánto grado ha contribuido los modelos o programas de este tipo al desarrollo de la nación?

3. ¿Qué hacer para revertir este proceso de masificación desplanificada en la gestión del Talento Humano en función del desarrollo sostenible y sustentable?

Por lo que implica poner en funcionamiento estructuras que se adecuen no sólo al momento histórico sino que articulen nuevas aproximaciones de efectividad, eficacia y eficiencia de las instituciones públicas vinculadas con políticas estratégicas que logren permear a la sociedad venezolana, de tal manera que se ejerza a través de ella la configuración entre lo estrecho establecido en la Constitución y el mundo circundante.

La gestión del Talento Humano responde a una política sistémica, un eje que transversaliza todo el proceso y que permite evidenciar cómo se organiza, direcciona y lleva a cabo la operatividad en términos de eficiencia y eficacia de un ente institucional. Sin embargo, cuando el carácter público se expresa en el manejo del estado, entendido este último como "la sociedad política" en lo relacionado a responder con la demanda de la población en todas las áreas en las que el gobierno tiene incidencia directa, y en el que los actores sociales (trabajadores y trabajadoras) que desarrollan tales tareas, forman parte de una: "organización o institución que logra traducir experiencias o necesidades de grupos sociales en programas de acción" para la construcción social del país y que le da fuerza a determinado proyecto político (Arzolay, 1998:14) sea este cual fuere. 


\section{Yoselin García Colina}

Lo anterior reviste importancia substancial en tanto las políticas públicas busquen convertirse en proyectos 0 actividades estatales para satisfacer las necesidades sociales, por lo que estas nociones apuntan al principal responsable en generarlas y mantenerlas: El estado, y las instituciones que lo administran, además como instrumento que fortalece el sistema político que lo compone, es decir, la acción de gobierno o el gobierno en acción (Oddone y Granato, 2008) y precisamente el talento humano funge como el brazo ejecutor permitiendo incidir tanto en el desarrollo humano de la población así como en lo institucional que garantice niveles de gobernabilidad.

Esa conjunción a las políticas de la agenda local que deben generarse desde los organismos públicos, resulta imperioso de la misma forma que también deben ser generadas otras que permeen directamente a quienes manejan y dirigen dichos procesos, he ahí la gran contradicción entre la macroplanificación y el abordaje de problemas y soluciones colectivas, pero que puedan engranarse con aquellos que tienen en sus manos la tarea de hacer efectiva la instrucción que se desprende posterior a la planificación.

Así que son las instituciones, en el mero sentido estricto y quienes operan las mismas, que deben garantizar que se atiendan con la mayor celeridad, eficacia y eficiencia las solicitudes de la población, en tanto en cuanto puedan crearse al mismo tiempo mecanismos de diligenciar con prontitud para que redunde en desarrollo y calidad de vida en todas las áreas, desde la educación, salud, transporte, vivienda, alimentación, etc., tanto para el público que está dirigido como para quien las dirigen; esto revierte mayor importancia cuando se determina que para que la política pública en las instituciones del estado se convierta en un factor estratégico para el desarrollo de una nación, se hace necesaria también la realización de una valuación pormenorizada de quienes y para quienes están siendo planificadas, dirigidas y ejecutadas.

En este sentido, la gestión del talento humano constituye no sólo el desarrollo de la institucionalidad sino que también coadyuva al desarrollo humano en términos del 


\section{Yoselin García Colina}

crecimiento profesional y por tanto personal. Sin embargo, las instituciones públicas venezolanas, y en particular en los entes regionales devienen de un sistema burocrático dependiente a la concentración de las decisiones como una forma de gerencia.

Estas presunciones no son para nada novísimas, sino que procuran la promoción e impulso de nuevo métodos que permitan, no como meras recetas, sino nuevas alternativas para posibilitar el inicio de la transformación efectiva, eficaz y eficiente de la clase trabajadora en el proceso social de trabajo y que directamente accione en ese sentido a las mejoras de las instituciones públicas al servicio de la sociedad, perfilados hacia lo humano y no hacia lo tecnócrata.

En este marco de ideas, el análisis que aquí se pretende busca corroer desde las bases los problemas que permean el ámbito institucional, en términos de acción gubernamental relacionado a la gestión de gobierno en cuánto a políticas públicas se refiere en lo general, pero esto precisamente en lo particular, deviene también a la gestión del talento humano para propiciar estos cambios de paradigmas que se orienten al desarrollo estratégico del país pero en la misma proporción como es necesario el desarrollo humano (PNUD, 1991).

La mayoría de las investigaciones acerca de las formas de abordaje de las contrariedades sobre el desarrollo subyacen en la perspectiva económica para lograr una explicación racional y causal de tales problemas de desigualdad en los países del mundo, y cómo tales males afectan el desarrollo humano. Por lo que se asume posturas como la de García Rabelo (et al., 2009) en donde destaca que:

Conscientes del reduccionismo economicista que lastró los estudios sobre el desarrollo durante mucho tiempo, (...) el subdesarrollo y, consecuentemente el desarrollo, son fenómenos multidimensionales, lo que equivale a considerar las dimensiones no sólo económicas, sino también las políticas, sociales, las culturales y ambientales. (p.5)

Lo anterior refiere señalar el hecho que deviene la evolución histórica de la Teoría Sobre la Transformación del Capitalismo- El capitalismo popular, El Estado de 


\section{Yoselin García Colina}

Bienestar, Capital Humano- en donde se evolucionara aceleradamente a nuevas sociedades más desarrolladas irrumpiendo en las discrepancias de clases (García Rabelo et al., 2009:9). Esto resalta las contradicciones de modelos que se contraponen al crecimiento de países que constituyen la cuarta parte poblacional del mundo.

Venezuela ha sido un ejemplo creación de un modelo alternativo que se contrapone a la lógica del capital, y que ha creado no sólo mecanismos de distribución equitativa de las riquezas a través de la creación del Sistema de Misiones y Grandes Misiones, sino que también ha permitido nuevas formas de participación y, por lo tanto nuevas formas de desarrollo con incidencias planetarias en las que se destacan a grandes rasgos, los avances en materia educativa, cumpliendo parte de las metas del milenio establecidas por la Asamblea General de la ONU (2000), entre otros tantos.

A la luz de estas consideraciones, se permite precisar que la gestión pública parte inicialmente del ser humano como núcleo que origina las transformaciones sociales que permitan avanzar en función del desarrollo alternativo, admitiendo a esta población desde lo local la resolución de problemas estructurales cónsonos con nuevos modelos de desarrollo social, económico y político. Esta consideración sobre la Teoría sobre Desarrollo Humano enmarcada en el Programa de las Naciones Unidas para el Desarrollo (1990:34), destaca tres niveles esenciales, como lo son: a) Disfrutar de una vida prolongada y saludable, b) Adquirir conocimientos y c) Tener acceso a los recursos necesarios para lograr un nivel de vida decente.

Esto hace imprescindible la gestión del talento humano como una política pública de perfeccionamiento del estado pero también de las posibilidades de potencializar y formar las capacidades y cualidades que permitan incorporar una perspectiva humanista con rasgos definitorios que identifique al ser humano con el hecho social de potenciación, productividad, sustentabilidad, cooperación, seguridad y equidad como motor del desarrollo. 


\section{Yoselin García Colina}

Esta mezcla de talento humano con la política pública de gestión de gobierno, resulta confusa si no se adoptan enfoques que permitan evidenciar desde la contextualización de las realidades sustentadas en las experiencias desde lo interno de las instituciones, como desde la perspectiva globalizada de los modelos que se han implementado en la administración pública y que vislumbra un sistema fijado en reproducir una forma de capitalismo que amplifique las oportunidades de crecimiento individual, sin medir las consecuencias de las desigualdades que produzca el proceso, tendiente a "generar un tipo de desarrollo humano, pero de manera restringida y condicionada, no universal, y nunca como estrategia" (García Rabelo et al., 2009:153).

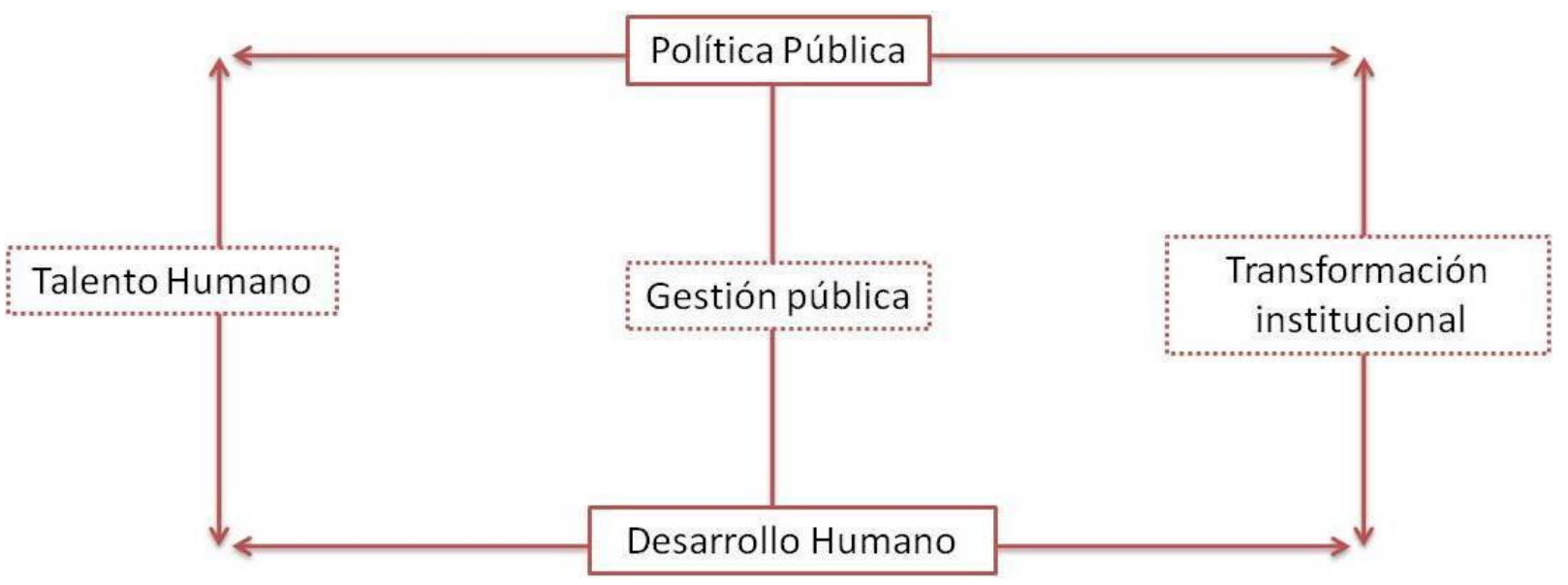

\section{Figura $\mathrm{N}^{\circ} \mathbf{1}$}

Pilares propuesto por la autora para un sistema de categorización enmarcado en un nuevo modelo de gestión del talento humano que motorice la gestión pública que permita mejorar la institucionalidad venezolana.

Esto produce en la gestión pública, una suerte de mercado intelectual que fluctúa entre las competencias que genera estas desigualdades y la forma de afectación del servicio estatal como parte de un proceso de desarrollo macro, que implica no sólo las probabilidades socio-económicas derivadas de la productividad del trabajo, sino las condiciones de socialización y política de "No-acaparamiento" del saber científico o 


\section{Yoselin García Colina}

inclusive experiencial que potencie las nuevas generaciones que apalancan la administración gubernamental, propiciando espacios de apropiación colectiva que entre el saber y el hacer.

Sin embargo, para potenciar modelos encaminados a deslastrar el rastro que el capitalismo ha dejado en la planificación del estado social y sobre todo en la formación del ser humano que describa nuevos valores y prácticas éticas, se encuentra un punto de contradicción entre las consideraciones medulares que recogen los informes del PNUD en tanto se distingan de la teoría de crecimiento económico de W. W. Rostow, que no abarque sólo un enfoque eminentemente pragmático sino que promueva políticas capaces de impulsar al talento humano desde el desarrollo humano. No obstante, para Hidalgo (2000):

Los postulados teóricos del pensamiento dominante, el neoliberal, sobre el que tan solo añaden matices sociales... lo cual no puede ser desligado del hecho de que los estudios sobre desarrollo humano están financiados por las Naciones Unidas donde el pensamiento neoliberal es el dominante (p.17).

Lo que implica, repensar nuevos abordajes teóricos y creación de nuevas metodologías que permitan avanzar en procura de la planificación de estructuras de pensamiento cónsonas con una nueva filosofía de actuación de los personajes que hacen vida en estos espacios institucionales para que sean perfectibles los patrones de comportamiento que circunscriben la esfera del sector público, como mecanismo indispensable para la transformación del Estado.

Para que una nación marche satisfactoriamente hacia el desarrollo económico y social, es necesario poner en relieve el desarrollo humano que redundará en la creación de políticas públicas más eficaces y eficientes. Sin embargo, para García Sánchez (2007), "comienza la preocupación por las ineficacias detectadas en la ejecución de los programas públicos, surgiendo el planteamiento de los fallos de la intervención del estado (...), asociados a la burocracia". Parte de esta afirmación se concentra en la determinación del principio de planificación, supervisión y acompañamiento que debe 


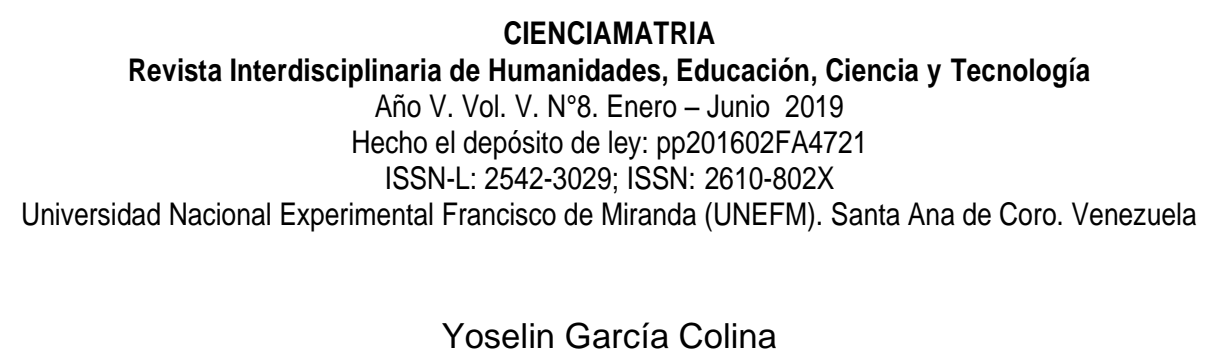

realizar el talento humano a la consecución de la política de gestión pública. Para Alburquerque (2002):

Una estrategia de desarrollo local se sustenta en tres pilares básicos como son la descentralización y el fortalecimiento de las administraciones locales; la creación de entornos territoriales innovadores; y el fomento de iniciativas locales de desarrollo económico local y generación de empleo y renta. (p.75)

Por lo que resalta a la nueva filosofía de gestión pública, utilizando palabras de Hood (1991, pp. 5-6), como "un matrimonio entre el Nuevo Institucionalismo y la gerencia profesional". Esta percepción puede resonar como más convencional y ortodoxa que el enfoque del desarrollo humano, sin embargo, la concepción de las dimensiones o sistema categoriales: Gestión-Talento Humano-Política Pública- Transformación institucional, orienta hacia un concepto más elevado que posibilite la trascendencia de un modelo desigual depredador hacia uno nuevo que contemple forjar y revalorizar una nueva cultura de servicio público consciente, comprometido, fundamentado en el principio del saber y del trabajo.

En este sentido, los aspectos a considerar para realizar aproximaciones lo más cercano de la realidad, deben gravitar en torno a la transformación social basada en la caracterización de la situación problémica, partiendo del principio que fundamenta la Investigación Interpretativa (Perafán, 2004) como modelaje de abordaje, pero definiendo estrategias coordinadas con prácticas sistémicas e integrales que consoliden un nuevo modelo de gestión al talento humano, en tanto se conciba como una política pública que coadyuve al desarrollo económico, social y humano de la nación con el propósito irrestricto de propiciar una institucionalidad que se robustezca en la misma medida un sistema de gobierno que tenga como centro de su naturaleza al ser humano.

\section{REFLEXIONES FINALES}

En Venezuela, la sociedad se ha vuelto hacia la institucionalidad como mecanismo de acercamiento a una nueva visión de gestión pública de gobierno que procura mitigar las 


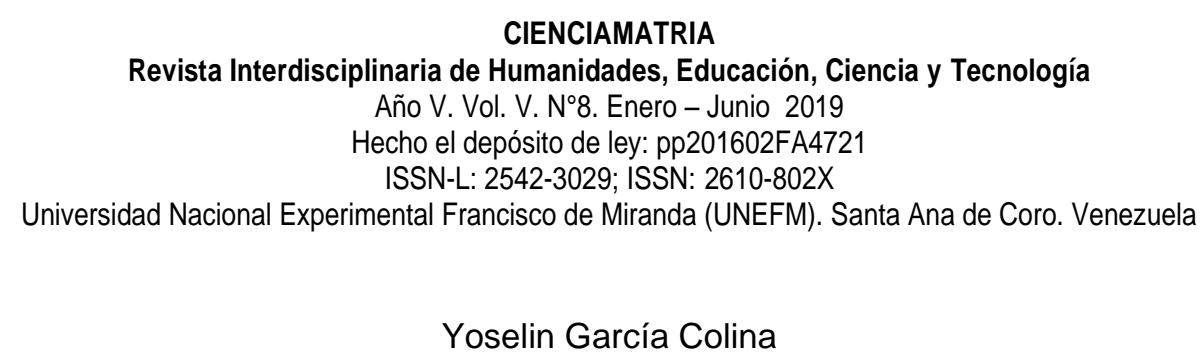

desigualdades en tanto se tengan las mismas oportunidades de acceder a la misma. Por lo que el talento humano que las dirige resulta un factor fundamental para el desarrollo y funcionabilidad de la institución que sirve como asentamiento de un sistema político y por lo tanto de políticas públicas que conlleven a minimizar las necesidades de los pueblos.

La afirmación anterior también destaca cómo se está concibiendo y repensando no sólo en el manejo y funcionamiento de las instituciones sino cómo está siendo planificado, encaminado y orientado el personal que la dirige.

Para responder a tales demandas resulta una tarea obligada la elaboración y ejecución de modelos organizacionales que respondan a la gestión de talento humano de una manera integrada, sistémica y estrechamente vinculada entre las necesidades colectivas de desarrollo y las individuales de crecimiento, para lograr mejores profesionales formados bajo este paradigma gerencial y contribuyendo a satisfacer las necesidades institucionales como motor en la administración y distribución de las riquezas del estado.

Esa nueva perspectiva de perfectibilidad debe ser profundizada y plasmada en los programas o planes de desarrollo de la nación, para que incluya al menos directrices que orienten a cementar las bases de la transformación permanente de la institucionalidad en la misma medida como se perfile al profesional que la administre.

Aunque resulte un proceso difícil de reversión lo imperioso en la aplicación de políticas públicas que se determinen y encaminen hacia los trabajadores y trabajadoras que hacen que las instituciones del estado estén a la altura de las demandas de las comunidades, que evalúan al sistema de gobernanza político, en parte, por la capacidad de respuesta de los entes y organizaciones que conduzca hacia el desarrollo sostenible y sustentable en el marco de sus potencialidades. 


\begin{abstract}
CIENCIAMATRIA
Revista Interdisciplinaria de Humanidades, Educación, Ciencia y Tecnología

Año V. Vol. V. N8. Enero - Junio 2019

Hecho el depósito de ley: pp201602FA4721

ISSN-L: 2542-3029; ISSN: 2610-802X

Universidad Nacional Experimental Francisco de Miranda (UNEFM). Santa Ana de Coro. Venezuela

Yoselin García Colina
\end{abstract}

\title{
REFERENCIAS CONSULTADAS
}

1. ALBURQUERQUE, F. (2002): "Guía para agentes. El desarrollo económico territorial". Sevilla: Instituto de Desarrollo Regional y Fundación Universitaria. España.

2. ALCÁNTARA, M. (1995): Gobernabilidad, crisis y cambio. México: Fondo de Cultura Económica.

3. ARZOLAY, C. (1998): "La Hegemonía de Acción Democrática en el Estado Monagas". Maturín, Venezuela.

4. CASTAÑEDA, L. (2014): "La Nueva Gestión Pública: Orientación Hacia El Rendimiento y una Administración Pública Progresiva". Universidad Militar Nueva Granada, Facultad de Economía, Especialización en Finanzas y Administración Pública. Bogotá, Colombia. Disponible en: http://repository.unimilitar.edu.co/bitstream/10654/12675/1/ENSAYO\%20DEFINIT IVO\%20LUIS\%20ORLANDO\%20CASTA\%C3\%91EDA\%20FIQUITIVA.pdf

5. CHÁVEZ, H. (2007): "El Libro Azul". Serie de folletos 4F. Ministerio del Poder Popular para la Educación.

6. CHIRINO, C., \& LAMUS DE RODRÍGUEZ, T. (2017). Modelo educativo para la participación comunitaria mediante el pensamiento crítico en la construcción de las comunas. Revista Arbitrada Interdisciplinaria Koinonía, 2(4), 110-131. Recuperado http://fundacionkoinonia.com.ve/ojs/index.php/revistakoinonia/article/view/72/59

7. GARCÍA, M. (2003): "El debate contemporáneo sobre el desarrollo y el subdesarrollo. Tendencias Actuales". Evento científico anual de la Facultad de Economía. IBSN 959.16-0244-8. Cuba.

8. GARCÍA, M., MUNSTER, B., LÓPEZ, C., HIDALGO, M., PICHS, R., RUÍZ, R. BARÓ, S., MEDINA, Z. (2009): "Las teorías acerca del subdesarrollo y el desarrollo: una visión crítica". Editorial Félix Varela. La Habana, Cuba.

9. GARCÍA SÁNCHEZ. I. (2007): "La nueva gestión pública: evolución y tendencias". Presupuesto y Gasto Público 47/2007: 37-64. Secretaría General de Presupuestos y Gastos. Universidad de Salamanca. Disponible en: http://www.ief.es/documentos/recursos/publicaciones/revistas/presu_gasto_publi co/47_GarciaSanchez.pdf 


\begin{abstract}
CIENCIAMATRIA
Revista Interdisciplinaria de Humanidades, Educación, Ciencia y Tecnología

Año V. Vol. V. N8. Enero - Junio 2019

Hecho el depósito de ley: pp201602FA4721

ISSN-L: 2542-3029; ISSN: 2610-802X

Universidad Nacional Experimental Francisco de Miranda (UNEFM). Santa Ana de Coro. Venezuela

Yoselin García Colina
\end{abstract}

10. GRANATO, L. \& ODDONE, C. (2008): "Nuevas propuestas de integración en América Latina: ALBA y Comunidad Sudamericana de Naciones". Buenos Aires. Universidad Abierta Interamericana.

11. HIDALGO, A. (2000): "El Concepto de Desarrollo Humano entendido como Negación de la Pobreza", ponencia presentada al evento Diez Años de Desarrollo Humano. Madrid.

12. HOOD, C. (1991): "A Public Management for all Seasons?". Reino Unido, Administración Pública. Vol. 69, núm. 1. pp. 3-19.

13. NÚÑEZ, P., \& OROZCO, J., (2013): "Las teorías del desarrollo en el análisis del turismo sustentable". InterSedes: Revista de las Sedes Regionales [en linea] 2013, XIV (Sin mes): [Fecha de consulta: 9 de marzo de 2017] Disponible en: http://www.redalyc.org/articulo.oa?id=66627452008

14. LEY DEL PLAN DE LA PATRIA (2013): Publicado en la Gaceta Oficial de la República Bolivariana de Venezuela №6.118. Caracas. Venezuela.

15. LÓPEZ, C. (2009): "Las teorías acerca del subdesarrollo y el desarrollo: una visión crítica". Colectivo de Autores. La Habana. Cuba.

16. OSBORNE, D. \& PLASTRIK, P. (1998): "La reducción de la burocracia: Cinco estrategias para reinventar el gobierno". España, Editorial Paidós.

17. PERAFÁN, G. (2004): "Epistemología del Profesor Universitario sobre su propio conocimiento profesional". Universidad Pedagógica Nacional. Volumen 1 de Colección Tesis Doctorales. Colombia.

18. PNUD (1990): "Informes sobre desarrollo humano". Ediciones Mundi Prensa, Madrid.

19. PNUD (1991): "Desarrollo humano: Informe 1991". Tercer mundo editores, S.A. Título Original: Human Development Report 1991. Traducción: Esperanza Meléndez y Ángela García. Bogotá, Colombia. Disponible en: http://biblioteca2012.hegoa.efaber.net/system/ebooks/1008/original/Informe_sobr e_Desarrollo_Humano_1991.pdf 
20.PRIETO, P. (2013): "Gestión del Talento Humano como Estrategia para retención del Personal". Universidad de Medellín, Facultad de Ciencias Económicas y Administrativas Especialización en Gestión del Talento Humano y la Productividad, Cohorte 32. Medellín, Colombia. Disponible en: http://repository.udem.edu.co/bitstream/handle/11407/160/Gesti\%C3\%B3n\%20d el\%20talento\%20humano\%20como\%20estrategia\%20para\%20retenci\%C3\%B3n $\% 20$ del\%20personal.pdf?sequence $=1$

21.RIST, G. (2002): "El desarrollo: historia occidental de una creencia". Instituto Universitario de Desarrollo y Cooperación, Universidad Complutense de Madrid. España.

C2019 por los autores. Este artículo es de acceso abierto y distribuido según los términos y condiciones de la licencia Creative Commons Atribución-NoComercial-Compartirlgual 4.0 Internacional (CC BY-NC-SA 4.0) (https://creativecommons.org/licenses/by-nc-sa/4.0/). 